\title{
Conception de Circuits MMIC sur GaAs Commandés par Voie Optique
}

\author{
S. Faci, C. Tripon-Canseliet, F. Deshours, C. Algani, G. Alquié, S. Formont* et J. Chazelas* \\ LISIF-UPMC, Case courrier 252 - 3, rue Galilée, 94200 Ivry/seine, France \\ * Thales Airborne Systems, Elancourt, France
}

\begin{abstract}
Résumé - L'utilisation de l'effet photoconducteur pour contrôler des circuits intégrés microondes est ici exploitée. Le contrôle est effectué sur un composant passif : un gap microruban photocommutateur qui présente l'avantage de pouvoir être intégré aisément dans une technologie de circuits MMIC industrielle.

Nous avons développé un modèle de photocommutateur nonlinéaire basé sur les mesures pour la conception d'un oscillateur MMIC à $5 \mathrm{GHz}$ fabriqué selon la technologie UMS PH25 sur GaAs. Cet oscillateur délivre un signal lorsque le gap, placé sur la source du transistor, est éclairé (état $\mathrm{ON}$ ) et ne fonctionne plus lorsque le gap n'est pas éclairé (état OFF). Ce fonctionnement repose sur la variation de l'impédance équivalente du gap en fonction de la puissance de l'éclairement. Les premiers tests de ce circuit opto-microonde sont présentés.
\end{abstract}

Mots clés: effet photoconducteur, gap microruban, oscillateur microonde.

\section{PRINCIPE DU CONTRÔLÉ OPTIQUE}

L'éclairement d'une discontinuité de ligne de transmission, microruban ou coplanaire, provoque un changement local des propriétés physiques du substrat semiconducteur dû à la création d'un plasma de paires électron-trou par absorption de photon. La nature de l'éclairement et la structure de la discontinuité permettent de réaliser diverses fonctionnalités microondes passives ou actives.

\section{A. Photocommutation microonde sous éclairement continu}

Les sources lasers utilisées délivrent des puissances variant entre 10 et 200 milliwatts afin de commuter des signaux continu ou microonde.

Le schéma d'un photocommutateur microruban classique est représenté sur la figure 1. En obscurité, la discontinuité atténue fortement la propagation d'un signal microonde injectée sur l'une de ces deux entrées. Sous éclairement continu, la couche plasma de porteurs libres photogénérée améliore la propagation du signal à travers la discontinuité. L'isolation et la transmission de ces dispositifs ne sont pas parfaites, une quantité complexe est définie pour les caractériser appelée rapport ON/OFF (1). Elle représente le rapport entre le coefficient de transmission sous éclairement à celui en obscurité.

$$
\Re_{\text {ON/OFF }}=\frac{S_{21}(\text { ON) }}{S_{21}(\text { OFF })}=R_{\text {ON/OFF }} e^{i \Delta \varphi_{\text {ON /OFF }}}
$$

A partir de cette entité et du circuit équivalent du photocommutateur, la structure de la discontinuité peut être optimisée pour ne faire varier qu'un paramètre à la fois, le module ou la phase de $R_{\text {ON/OFF }}$. En faisant varier le module uniquement, nous pouvons réaliser des fonctions de commutation ou d'atténuation. Si la phase uniquement change par excitation laser, la fonction réalisée est le déphasage microonde. Dans le cas le plus général, le module et la phase de Ron/OFF varient.

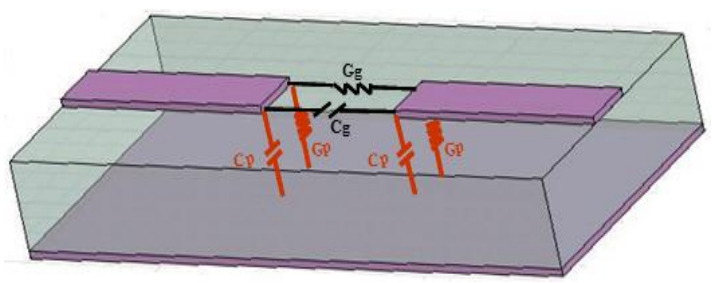

Fig. 1: Photocommutateur microruban et son circuit électrique équivalent

\section{B. Excitation optique pulsée}

Ces applications ultra-rapides reposent sur l'utilisation de lasers à impulsion femtoseconde, qui créent quasiinstantanément des distributions de porteurs hors équilibre en utilisant des énergies de l'ordre du Mégawatts.

L'application majeure est l'échantillonnage qui consiste en la génération des signaux picosecondes.

\section{MODELISATION DU PHOTOCOMMUTATEUR CLASSIQUE - ETAT DE L'ART}

\section{A. Modélisation électrique en obscurité}

Le modèle électrique du photocommutateur en technologie microruban, réalisé sur un substrat semiconducteur de haute résistivité, est constitué d'une capacité de couplage $\mathrm{Cg}$ et de deux capacités parallèles $\mathrm{Cp}$ en configuration symétrique $\pi$ (figure 1).

Plusieurs travaux basés sur le calcul de la densité de charge des lignes de transmission ont été effectués [1, 2, 3] et ont abouti à des expressions de capacités dépendant des dimensions du gap, de la constante diélectrique et de la hauteur du substrat semiconducteur. 


\section{B. Modélisation sous éclairement}

La couche plasma créée sous éclairement continu est caractérisée physiquement par une conductivité constante $\Delta \sigma_{\mathrm{m}}$ (2) et une profondeur $d_{e}$. Cette conductivité est proportionnelle à la densité de porteurs photogénérés qui dépend des paramètres du faisceau lumineux (puissance et longueur d'onde) et des paramètres intrinsèques $\mathrm{du}$ substrat semiconducteur [4].

$$
\begin{aligned}
& \Delta \sigma_{m}=\frac{\Delta \sigma_{p h}}{(1+\alpha L)}\left[\frac{1}{\alpha}\left(\frac{\alpha L^{2}+v_{s} \tau}{v_{s} \tau+L}\right)\right]^{\frac{-\alpha L}{(1-\alpha L)}} \\
& \Delta \sigma_{p h}=\frac{e}{h v}\left(\mu_{n}+\mu_{p}\right) \eta \alpha \tau(1-R) \frac{P_{o p}}{A}
\end{aligned}
$$

Le modèle électrique $\mathrm{du}$ photocommutateur sous éclairement continu est identique à celui en obscurité à l'exception de la présence d'une conductance série Gg (4) obtenue en intégrant la conductivité $\Delta \sigma_{\mathrm{m}}$ sur les dimensions de la couche plasma.

Pour un éclairement impulsionnel, un modèle électrique a été développé dans le domaine temporel [5] qui tient compte $\mathrm{du}$ dopage du substrat et la dynamique des porteurs. La photogénération de paires électron-trou est synthétisée par deux sous-circuits qui modélisent chacun la variation temporelle de la densité de porteurs libres satisfaisant les équations de continuité dans le domaine temporel. Une nouvelle expression de la photoconductance (5) avec dépendance temporelle a été établie.

$$
\begin{gathered}
G_{g}=\frac{W_{e f f} \Delta \sigma_{h}}{L_{g}\left(1-\alpha^{2} L^{2}\right)}\left[\frac{1}{\alpha}-L \frac{\alpha L^{2}+v_{s} \tau}{v_{s} \tau+L}\right] \\
G_{g}(t)=\frac{q L_{g} W_{g}}{d_{e}}\left[\mu_{n}\left(n_{0}+V_{n}(t) \cdot 10^{b}\right)+\mu_{p}\left(n_{0}+V_{p}(t) \cdot 10^{b}\right)\right]
\end{gathered}
$$

\section{Modélisation électromagnétique}

Les simulateurs électromagnétiques $2.5 \mathrm{D}$ et $3 \mathrm{D}$ sont très utilisés pour la modélisation sous éclairement des composants opto-microonde. Ils permettent aussi de caractériser les plots de test (transition microruban-coplanaire) manquants dans les simulateurs électrique et électromagnétique.

Les simulateurs 3D permettent de simuler particulièrement les couches minces et ont l'avantage de flexibilité par rapport aux logiciels 2.5D. En effet, la couche de plasma photogénérée dans un semiconducteur GaAs, a une épaisseur de quelques microns et est facilement paramétrable en profondeur et en surface.

L'effet photoconducteur est modélisé par une couche de conductivité constante de valeur $\Delta \sigma_{\mathrm{m}}$ et de profondeur $\mathrm{d}_{\mathrm{e}}$, sa surface est estimée à partir du diamètre de la tache lumineuse. Dans le cas où l'éclairement du semiconducteur est assuré par une fibre optique, la surface de la couche plasma est calculée en fonction du diamètre de la fibre et de sa distance par rapport à la surface semiconductrice éclairée.

\section{MODELISATION NON-LINEAIRE À PARTIR DES MESURES DE PARAMETRES-S}

\section{A. Etude qualitative de la permittivité}

L'application d'un champ électrique d'origine optique sur un substrat semiconducteur crée une polarisation locale parallèle à ce champ. Il en résulte une modification locale de la permittivité qui dépend essentiellement du coefficient d'absorption inter-bande $\alpha(\lambda)$ propre au matériau et de la longueur d'onde $\lambda$ du champ appliqué. Pour les fréquences optiques et les caractéristiques des substrats semiconducteurs usuels, la permittivité de la couche plasma induite reste inchangée. La conductance ainsi créée (4) varie seulement en fonction de la longueur d'onde $\lambda$ et de la puissance de l'onde optique.

Par contre, la propagation d'un signal microonde dans un plasma de paires électron-trou influe sur ces paramètres physiques. Sa permittivité devient complexe $[6,7]$ et est exprimée par (6). Elle dépend fortement de la densité de porteurs excédentaires et de la fréquence du signal microonde.

$$
\begin{gathered}
\varepsilon=\tilde{n}^{2}=\varepsilon_{r}-\sum_{k=n, p} \frac{w_{p k}{ }^{2}}{w^{2}+v_{k}^{2}}-i \sum_{k=n, p} \frac{w_{p k}{ }^{2} v_{e}}{w\left(w^{2}+v_{k}^{2}\right)}=\varepsilon^{\prime}-i \varepsilon^{\prime \prime} \\
w_{p k}=\frac{\Delta n e^{2}}{\varepsilon_{0} m_{k}^{*}} ; v_{k}=\frac{e}{\mu_{k} m_{k}^{*}}
\end{gathered}
$$

La variation de la permittivité pendant l'éclairement rend les expressions de la capacité $\mathrm{Cg}$ et de la conductance $\mathrm{Gg}$ invalides, ces deux éléments doivent varier avec la fréquence du signal microonde. Pour valider et vérifier cette dépendance fréquentielle, nous avons comparé la simulation du circuit équivalent $\mathrm{du}$ photocommutateur avec les mesures en obscurité et sous éclairement. Ensuite, nous avons extrait ces différents paramètres à partir des mesures, les résultats obtenus sont présentés ci-après.

\section{B. Mesures du photocommutateur}

Des mesures sous pointes ont été effectuées sur différents types de photocommutateurs sur GaAs dans le domaine fréquentielle 0.1-40 GHz. La longueur d'onde de la source laser utilisée est égale à $671 \mathrm{~nm}$ et délivre une puissance maximale de l'ordre de $130 \mathrm{~mW}$.

Nous avons retenu pour illustration les réponses d'un photocommutateur à géométrie rectangulaire de longueur $50 \mu \mathrm{m}$. La figure 2 représente le module et la phase du coefficient de transmission en obscurité et sous éclairement avec différents niveaux de puissance optique.

En éclairant le gap, la transmission du signal est améliorée et sa phase diminue qui est due à la photogénération de paires électron-trou, ces variations dépendent de la puissance optique injectée et de la fréquence du signal radiofréquence. Ainsi, pour ce photocommutateur le module et la phase du rapport ON/OFF varient. 


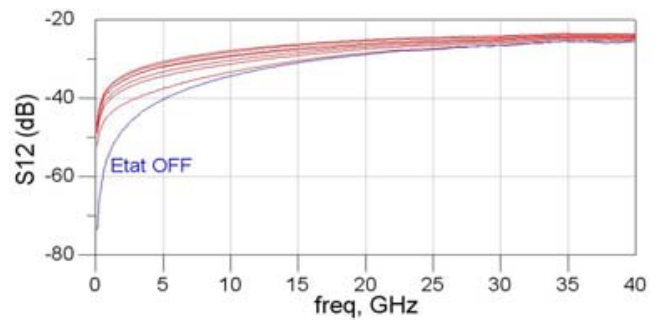

(a)

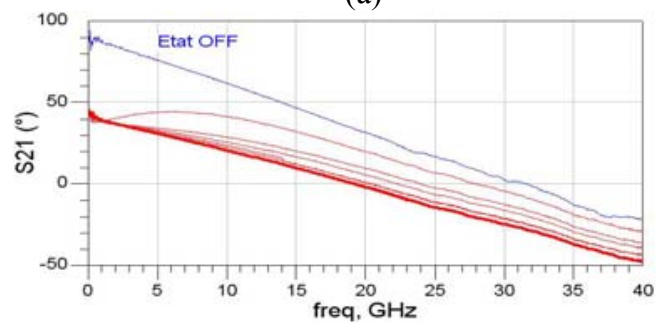

(b)

Fig. 2: Mesures du coefficient de transmission en a) amplitude et - b) en phase du photocommutateur en obscurité et pour différents niveaux de puissance optique

La figure 3 représente une comparaison entre la simulation $\mathrm{du}$ circuit équivalent du photocommutateur et les mesures en obscurité et sous éclairement avec une puissance de $120 \mathrm{~mW}$. En obscurité l'écart entre la simulation et l'expérimentation sur toute la bande fréquentielle est assez faible. Par contre sous éclairement cet écart devient très important pour le module et la phase du coefficient de transmission. La même constatation est obtenue en modifiant la puissance optique et avec une autre longueur du gap.
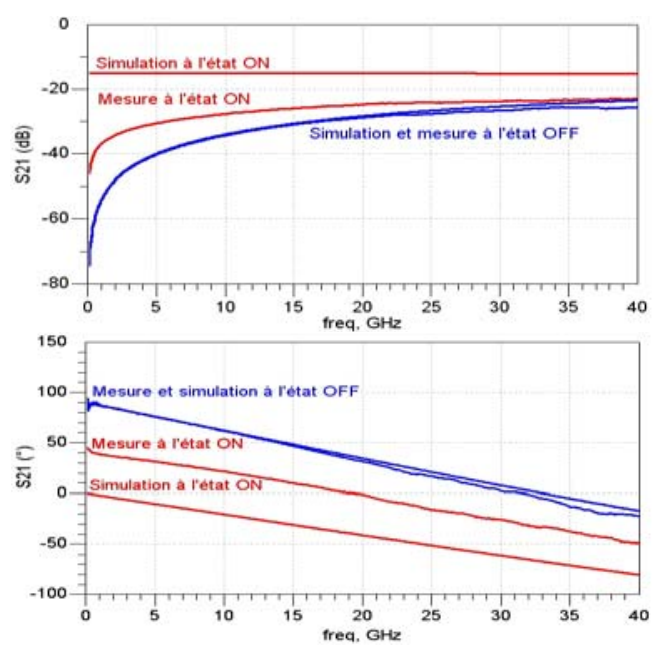

Fig. 3: Mesure et simulation du coefficient de transmission du photocommutateur en obscurité et sous une puissance de $120 \mathrm{~mW}$

\section{Extraction des paramètres électriques}

L'extraction des différents paramètres du circuit équivalent $\mathrm{du}$ photocommutateur est obtenue par identification de sa matrice impédance avec la matrice des paramètres $\mathrm{S}$ issue des mesures, les résultats obtenus sont représentés sur la figure 4 . Nous observons trois points essentiels:

- les valeurs des éléments extraits en obscurité présentent un écart faible par rapport aux valeurs théoriques;

- les éléments parallèles extraits en obscurité et sous éclairement sont quasi-identiques quelques soient la puissance injectée;

- la non-linéarité des éléments séries extraits en fonction de la fréquence microonde. Ces éléments séries sont modulés par la puissance optique appliquée.

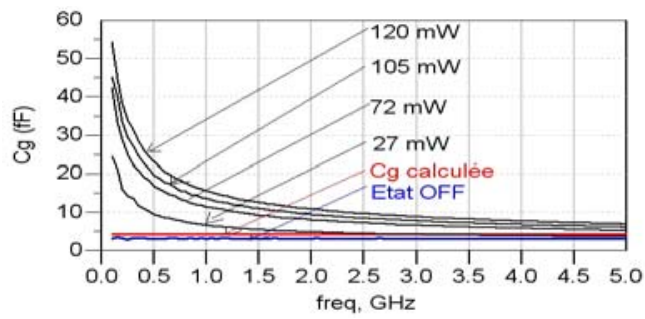

(a)

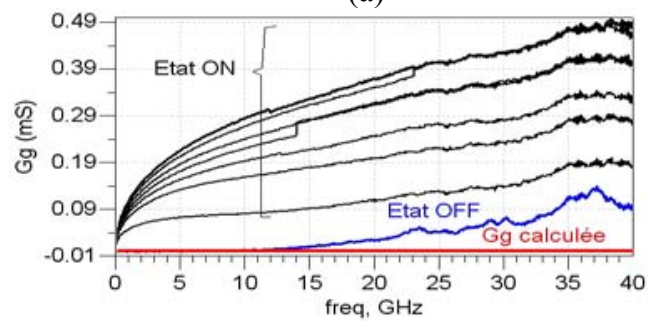

(b)

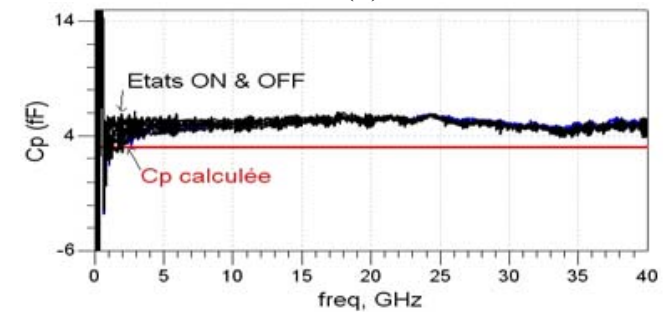

(c)

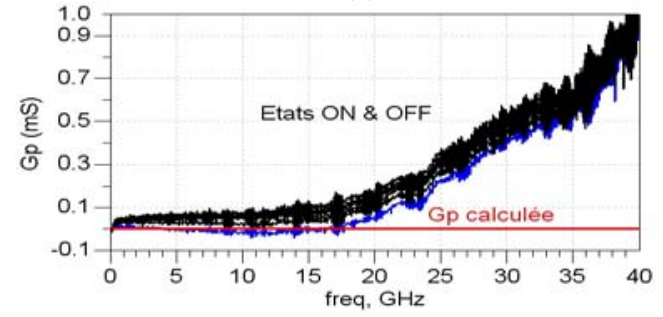

(d)

Fig. 4: Extraction des paramètres électriques du photocommutateur, a) capacité série, b) conductance série, c) capacité parallèle, d) conductance parallèle

D'après ces deux précédents résultats, la modélisation électrique classique du photocommutateur n'est valide qu'en obscurité. Les expressions de la photoconductance (4) et de la 
capacité de couplage donnée dans [1] ne sont plus valables dans l'état éclairé en présence d'un signal radiofréquence. Ces éléments séries du photocommutateur ont un comportement non-linéaire en fonction de la fréquence microonde. Il est nécessaire de les redéfinir en fonction de la fréquence de ce signal, des paramètres de l'onde optique et des caractéristiques intrinsèques du substrat semiconducteur pour pouvoir les intégrer dans des logiciels de simulation commerciaux.

\section{PRINCIPE DE L'OSCILLATEUR CONTRÔLÉ OPTIQUEMENT}

\section{A. Principe de fonctionnement}

Notre objectif est de réaliser un oscillateur MMIC sur GaAs à $5 \mathrm{GHz}$ fonctionnant seulement à l'application d'un signal optique de puissance et de longueur d'onde adéquates. Ce contrôle optique sur le circuit est appliqué sur un photocommutateur de longueur $50 \mu \mathrm{m}$. Ce gap présente des impédances différentes pour chaque état d'éclairement que nous exploitons dans l'étude de la stabilité d'un transistor PHEMT de longueur de grille $0.25 \mu \mathrm{m}$ de la fonderie UMS.

\section{B. Etude de la stabilité}

Un oscillateur microonde est constitué de 03 blocs schématisés sur la figure 5. Les étapes de conception de l'oscillateur sont résumées ci-dessous:

- choisir une configuration qui rend le transistor instable à la fréquence de fonctionnement (le facteur de Rollet soit inférieur à l'unité);

- choisir une impédance de charge $\mathrm{Z}_{\mathrm{L}}$ pour laquelle le module du coefficient de réflexion en entrée du transistor soit supérieur à un;

- fixer la fréquence d'oscillation avec un résonateur calculé à partir de la condition d'oscillation.

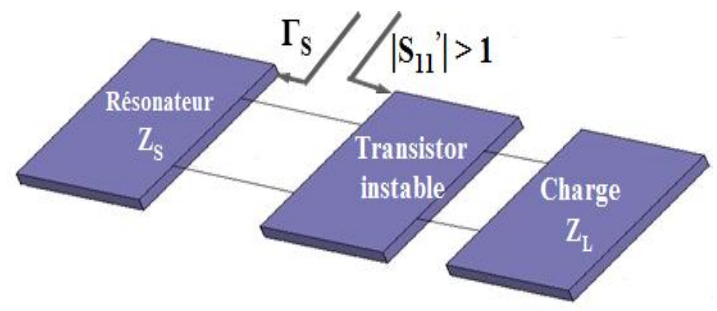

Fig. 5 : Schéma synoptique d'un oscillateur

La conception de l'oscillateur commandé optiquement suit les mêmes étapes précédentes et le photocommutateur doit être intégré dans l'un des trois blocs constituant l'oscillateur. L'impédance du photocommutateur est très grande en obscurité et elle reste assez élevée sous éclairement, nous ne pouvons l'intégrer pour synthétiser l'impédance de charge ou l'impédance du résonateur. Le photocommutateur est finalement inséré dans le bloc du transistor.

Pour avoir un fonctionnement de l'oscillateur uniquement sous éclairement, l'idéal est de choisir une configuration transistor-photocommutateur pour laquelle le système est instable à l'état $\mathrm{ON}$ et stable à l'état OFF. Cette solution n'a pas été accomplie avec le transistor et la longueur du gap que nous disposons.

L'autre solution consiste à choisir une impédance de charge pour laquelle le circuit est en instabilité maximale [8] à l'état $\mathrm{ON}$, peu importe qu'il soit instable ou non à l'état OFF. En d'autre terme, choisir une impédance de charge pour laquelle le module du coefficient d'entrée du circuit à l'état ON est supérieur à celui de l'état OFF. Un choix judicieux du résonateur permet de fixer la fréquence d'oscillation et de garantir les oscillations seulement à l'état $\mathrm{ON}$. Pour obtenir le facteur de Rollet du système inférieur à un, le gap est inséré entre la source du transistor et la masse en configuration source-commune.

\section{Conditions d'oscillations}

Les conditions d'oscillations d'un oscillateur classique sont exprimées par les trois formules suivantes:

$$
\begin{gathered}
\operatorname{Re}\left(Z_{S}\right)<\mid \operatorname{Re}(\text { Zin }) \mid \\
\left\{\begin{array}{l}
\operatorname{Re}\left(Z_{S}\right)+\operatorname{Re}\left(Z_{i n}\right)=0 \\
\operatorname{Im}\left(Z_{S}\right)+\operatorname{Im}\left(Z_{i n}\right)=0
\end{array}\right.
\end{gathered}
$$

L'expression (6) représente la condition de déclenchement des oscillations ; la résistance du résonateur est choisie à partir du module de la résistance négative petit-signal d'entrée du transistor chargé par $\mathrm{Z}_{\mathrm{L}}$. L'expression (7) permet de calculer la fréquence d'oscillation à partir de la réactance de l'impédance d'entrée du circuit.

Pour l'oscillateur contrôlé par voie optique, la condition (6) est restreinte. La résistance du résonateur est choisie entre le module de la résistance négative petit-signal d'entrée du circuit à l'état $\mathrm{ON}$ et celle à l'état OFF (8). Avec cette condition toute forme d'oscillation en obscurité est inexistence. La fréquence d'oscillation est obtenue à partir de l'expression (9).

$$
\begin{gathered}
\left\{\begin{array}{c}
\operatorname{Re}\left(Z_{S}\right)<\mid\left.\operatorname{Re}(\text { Zin })\right|_{\text {ON }} \\
\operatorname{Re}\left(Z_{S}\right)>\mid\left.\operatorname{Re}(\text { Zin })\right|_{\text {OFF }}
\end{array}\right. \\
\left\{\begin{array}{c}
\operatorname{Re}\left(Z_{S}\right)=\mid\left.\operatorname{Re}(\text { Zin })\right|_{\text {ON }} \\
\operatorname{Im}\left(Z_{S}\right)+\operatorname{Im}(\text { Zin })_{\text {ON }}=0
\end{array}\right.
\end{gathered}
$$

\section{RESULTATS DE SIMULATION ET DES MESURES}

\section{A. Résultats de simulation}

La simulation fréquentielle à l'état $\mathrm{ON}$ a délivré une sinusoïde de haute pureté spectrale à $4.985 \mathrm{GHz}$, la puissance 
de sortie est de l'ordre de $-7.2 \mathrm{dBm}$. Le niveau des harmoniques est très faible par rapport à la fréquence fondamentale. A l'état OFF, nous n'avons obtenu aucune forme d'oscillation.

Une variation de la puissance optique injectée, modélisée par une impédance de gap différente, entraîne une variation de la fréquence d'oscillation. Cette plage d'accordabilité $\Delta \mathrm{f}$ est de l'ordre de $60 \mathrm{MHz}$ pour une variation de puissance optique $\Delta \mathrm{p}$ de $50 \mathrm{~mW}$ (figure 6). Cet intervalle d'accordabilité peut être augmenter en modifiant l'impédance de charge, l'impédance du résonateur et la puissance optique injectée.

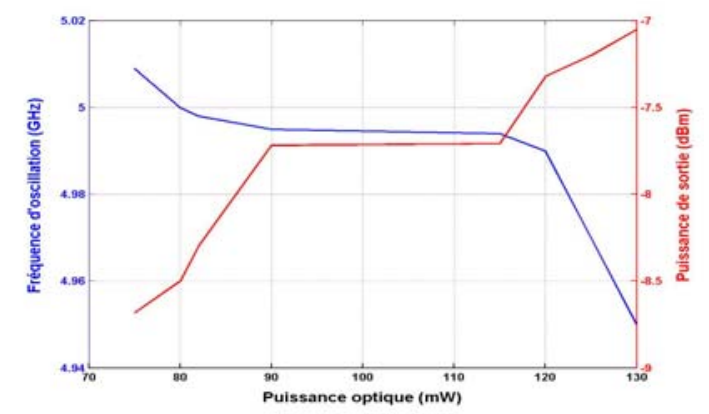

Fig. 6: Variation de la fréquence et de la puissance de sortie en fonction de la puissance optique injectée

\section{B. Résultats de mesures}

La figure 7 représente la réponse du circuit à l'application d'un signal optique de puissance $120 \mathrm{~mW}$ mesurée par un analyseur de spectre. Les oscillations disparaissent complètement en obscurité et elles reprennent une fois que la lumière est appliquée.

L'intervalle de variation de la fréquence d'oscillation en fonction de la puissance optique injectée n'a pas été obtenu, seulement une variation de quelques mégahertz a été mesurée. Ceci est dû à plusieurs paramètres dont les variations technologiques.

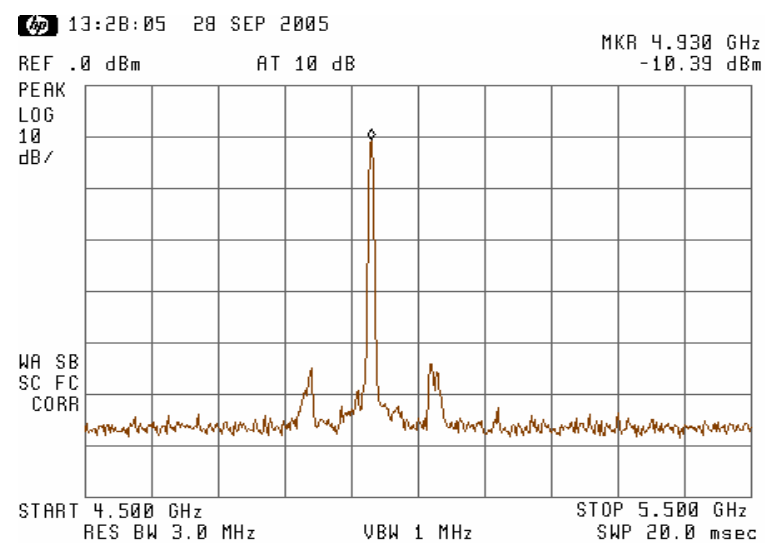

Fig. 7: Réponse de l'oscillateur sous un éclairement de puissance $120 \mathrm{~mW}$

\section{CONCLUSION}

Pour la conception de l'oscillateur commandé optiquement, nous avons exploité les mesures du photocommutateur en fonction de la puissance optique et de la fréquence microonde. Cela en raison de l'invalidité des expressions théoriques des éléments séries du gap sous éclairement en présence d'un signal radiofréquence. La dépendance fréquentielle de ces éléments est en cours d'étude.

La commande optique est appliquée sur un photocommutateur de longueur $50 \mu \mathrm{m}$ par le biais d'une fibre optique alimentée par une diode laser de longueur d'onde 671 nm. L'oscillateur est mis en marche à l'application d'une puissance optique de $120 \mathrm{~mW}$ délivrant une sinusoïde à 4.93 $\mathrm{GHz}$, en obscurité les oscillations s'évanouissent.

Les temps de commutation ON/OFF et OFF/ON de l'oscillateur seront déterminés par les mesures et notamment l'influence d'une commande optique impulsionnelle rapide sur la réponse du circuit. Ceci nous permettra de caractériser l'influence du photocommutateur sur les transitoires de ce circuit fortement non linéaire.

Le photocommutateur est très souple à intégrer ainsi d'autres conceptions de circuits MMIC actifs sont également envisagées.

\section{BIBLIOGRAPHIE}

[1] P. Benedek et P. Silvester, "Equivalent capacitance for microstrip Gaps and Steps", IEEE Transactions on Microwave Theory and Techniques, Vol. MTT-20, No. 11, November 1972

[2] A Farrar et A.T. Adams, "Matrix Methods for Microstrip Three-Dimensional problems", IEEE Transactions on Microwave Theory and Techniques, Vol. MTT-20, No. 8, August 1972

[3] R. Garg et I.J. Bahl, "Characteristics of coupled Microstriplines", IEEE Transactions on Microwave Theory and Techniques, Vol. MTT-27, No. 7, July 1979

[4] W. Platte et B. Sauerer, "Optically $C W$-included losses in semiconductor planar waveguides", IEEE Transactions on Microwave Theory and Techniques, Vol. 37 No. 12, pp139-148, January 1989

[5] C. Canseliet, "Commande optique des circuits microondes, Applications à la modulation et à l'échantillonnage", thèse de doctorat de l'université Pierre et Marie Curie (Paris)

[6] M. Fox, "Optical properties of solids", Oxford University press, 2001

[7] Chi H. Lee, "Picosecond optics and microwave technology", IEEE Transactions on Microwave Theory and Techniques, Vol. 38 No. 5, May 1990

[8] C. Gentili, "Amplificateurs et oscillateurs microondes", Masson, 1994 